\title{
Integrating Fitness Components Reveals That Survival Costs Outweigh Other Benefits and Costs of Group Living in Two Closely Related Species
}

\author{
Lyanne Brouwer, ${ }^{1,2,3, *, \dagger}$ Andrew Cockburn, ${ }^{3}$ and Martijn van de Pol $^{2, \dagger}$
}

1. Department of Animal Ecology and Physiology, Institute for Water and Wetland Research, Radboud University, Nijmegen, The Netherlands; 2. Department of Animal Ecology, Netherlands Institute of Ecology (NIOO-KNAW), Wageningen, The Netherlands; 3. Division of Ecology and Evolution, Research School of Biology, Australian National University, Canberra, Australian Capital Territory 2601, Australia

Submitted March 26, 2019; Accepted July 15, 2019; Electronically published January 6, 2020

Online enhancements: appendix. Dryad data: https://doi.org/10.5061/dryad.92rv6bq.

\begin{abstract}
Aвstract: Group living can be beneficial when individuals reproduce or survive better in the presence of others, but, simultaneously, there might be costs due to competition for resources. Positive and negative effects on various fitness components might thus counteract each other, so integration is essential to determine their overall effect. Here, we investigated how an integrated fitness measure (reproductive values [RVs]) based on six fitness components varied with group size among group members in cooperatively breeding red-winged and superb fairy wrens (Malurus elegans and Malurus cyaneus, respectively). Despite life-history differences between the species, patterns of RVs were similar, suggesting that the same behavioral mechanisms are important. Group living reduced RVs for dominant males, but for other group members, this was true only in large groups. Decomposition analyses showed that our integrated fitness proxy was most strongly affected by group size effects on survival and was amplified through carryover effects between years. Our study shows that integrative consideration of fitness components and subsequent decomposition analysis provide much needed insights into the key behavioral mechanisms shaping the costs and benefits of group living. Such attribution is crucial if we are to synthesize the relative importance of the myriad group size costs and benefits currently reported in the literature.
\end{abstract}

Keywords: cooperative breeding, Malurus, survival, reproductive value, decomposition analysis, nonadditive effects.

\footnotetext{
* Corresponding author; email: lyanne@myscience.eu.

$\dagger$ These authors served as lead authors for the project and contributed equally.
}

ORCIDs: Brouwer, https://orcid/org/0000-0001-6728-4851; Cockburn, https://orcid/org/0000-0002-8531-3350; van de Pol, https://orcid/org/0000 -0003-4102-4079.

Am. Nat. 2020. Vol. 195, pp. 201-215. (c) 2020 by The University of Chicago. 0003-0147/2020/19502-59147\$15.00. All rights reserved.

DOI: $10.1086 / 706475$

\section{Introduction}

Individuals often derive benefits from living in groups but may also suffer costs derived from the presence of others. Individuals may survive or reproduce better in groups, for example, because living in groups can increase foraging success (Stander 1992), facilitate thermoregulation (Gilbert et al. 2009), and provide protection against predators (Hamilton 1971; Sorato et al. 2012). Sometimes group members even assist in raising each other's offspring (Brown 1987; Koenig and Dickinson 2016), which can increase current reproduction (Emlen and Wrege 1991; Koenig and Walters 2011) or reduce the workload and thereby increase subsequent parental survival (Meade et al. 2010; Paquet et al. 2015). However, group members can also affect fitness negatively, as the presence of additional group members can increase intraspecific competition for resources (Newton 1992), which might result in increased stress (Markham et al. 2015), reduced mating opportunities (Heg et al. 2008; Huchard and Cowlishaw 2011), and reduced survival (Brouwer et al. 2006). Furthermore, fitness may vary with group size in a nonlinear way, such that smaller groups may benefit from additional group members, whereas larger groups may suffer a cost (Packer et al. 1988).

A limitation in the literature is that many studies on group living quantify fitness costs and benefits on only a single fitness component, particularly reproductive success (Stacey and Koenig 1990; Ebensperger et al. 2012; Koenig and Dickinson 2016). However, those studies that do consider multiple fitness components suggest that in many group-living species there is evidence that various costs and benefits co-occur (e.g., Chapman and Chapman 2000; Majolo et al. 2008), which may not be surprising given the many mechanisms via which group members might affect each other during different parts of the life 
cycle. Effects of group size on one component of fitness might thus not be representative for the effect that group size has on overall fitness. Furthermore, some effects might be more important than others. For example, fitness in long-lived species is generally much more sensitive to variation in adult survival (determining the number of lifetime breeding attempts) than to annual reproductive output (Clutton-Brock 1988; Newton 1989). In such situations, large positive effects of group size on reproduction can be easily outweighed by small — statistically hard to detectnegative effects on the survival of adults. In addition, group size effects on one fitness component may not be independent of those on another fitness component, and the costs and benefits of group living may thus not be additive. For example, positive effects of group size on reproductive success may contribute strongly to overall fitness only if there are no negative effects of group size on offspring survival.

To fully understand how overall fitness varies with group size, we first need to combine multiple fitness components for individuals living in various group sizes into an integrated fitness measure-something that has not been done before, as far as we are aware. Second, we need to quantify how the effect of group size on specific fitness components contributes to how an integrated fitness measure depends on group size, such that we gain a better understanding of the most important underlying behavioral mechanisms shaping the costs and benefits of group living. Finally, we need to quantify this for different types of group members, as individuals of different social statuses and sexes are expected to differ in the costs and benefits of group living (e.g., Clutton-Brock et al. 2006), such that the fitness landscape is likely to vary across the entire range of group sizes for all types of group members.

Here, we combine six separate fitness components into one aggregate fitness proxy, using the concept of reproductive value (RV; Fisher 1930). RV describes the expected contribution by individuals of a given age or stage class (here, group size, sex, and social status) to the future longterm growth of a population. Although RV is not equivalent to fitness in the sense of the change in frequency of an allele, it can be seen as a useful integrated fitness proxy that can inform us about optimal life-history strategies (Fisher 1958; Caswell 1982; Goodman 1982; Horn and Rubenstein 1984) and the strength of selection that acts on behavioral decisions that alter group size (Mumme et al. 1989). RVs are particularly suitable for species with a distinct age- or stage-structured life cycle, such as cooperative breeders. The reason is that in such species, the annual contribution of an individual in a given stage class to fitness is not a simple sum of the number of gene copies contributed to the next generation (year), because a breeder that survives to the next year (residual RV) is worth more than a surviving offspring that will most likely become a subordinate the next year (current RV).

Another advantage of using this mathematical description of fitness is that it allows us to use standard demographic modeling tools to perform decomposition analysis. This means that we can quantify for the first time how the effect of group size on one fitness component contributes to the effect of group size on a proxy of overall fitness, and we can do so for different types of group members (e.g., males, females, dominants, subordinates). Furthermore, by perturbing the effects of group size either on a single fitness component or on multiple fitness components, we can specifically test whether group size effects on fitness components interact with each other in a nonadditive way to determine overall fitness effects, which is an unexplored area of research. Our framework is general and can easily be modified to any group-living species, even if fitness components are not measured across the entire lifetime of individuals. Such analysis will ultimately help to determine what types of behavioral mechanismsout of the myriad effects currently reported in the literature - are most important in shaping the evolution of group living.

Our model species are group-living and cooperatively breeding superb fairy wrens (SFWs; Malurus cyaneus) and red-winged fairy wrens (RWFWs; Malurus elegans). These species are very similar in their ecology and many aspects of social organization. In both congeneric species, males are highly philopatric and usually stay in their natal territory for at least 1 year to assist the dominant pair (Rowley and Russell 1997). Most males spend their entire life on their natal territory or disperse to a breeding vacancy nearby (Russell and Rowley 2000; Cockburn et al. 2008). However, the two species differ in some key aspects of their life histories. First, whereas female offspring also delay dispersal to help in RWFWs, there is obligate female dispersal in SFWs, and, generally, only those that obtain a dominant position on a foreign territory are able to survive to the next breeding season (Rowley et al. 1988; Cockburn et al. 2003). As a result, SFWs virtually never have female subordinates, and their group sizes are 1.5 times smaller compared to RWFWs (where both sexes routinely help; see "Results"). Second, despite both being long-lived, SFWs have a lower survival than RWFWs (Cockburn et al. 2008; Lejeune et al. 2016), resulting in a twofold-lower life expectancy (see "Results"). Furthermore, RWFWs have a much (s)lower reproductive rate, as they only rarely rear two broods to independence in a season, whereas the longer breeding season of SFWs means that females of this species can raise three broods to independence (Russell and Rowley 2000; Cockburn et al. 2016).

We predict that RVs vary nonlinearly with group size because initial benefits of larger group size might be 
outweighed by increased competition. Furthermore, we predict that how RVs depend on group size will vary among different types of group members, because in both species, dominant males lose reproductive success to subordinates (fairy wrens are highly promiscuous; Double and Cockburn 2003; Brouwer et al. 2011), whereas dominant females gain survival benefits from having subordinates (at least in SFWs; Russell et al. 2007). Since a "fast" life history should result in a greater emphasis on current rather than future reproduction (Stearns 1983), the long life spans of both species suggest that effects of group size on survival will be a major factor determining the overall effect of group size on RVs. Yet the faster life history of SFWs should result in group size effects on reproductive success being relatively more important compared to RWFWs. Finally, the extreme philopatry means that there can be carryover effects of group size from one year to the next. For example, individuals in larger groups might reproduce or survive better and consequently may be more likely to live in large groups again the next year and reap additional benefits. Here, we explicitly model these transition probabilities, allowing estimation of the carryover effects of group size.

\section{Methods \\ Data Collection}

RWFW data were collected in Smithbrook Nature Reserve in Western Australia $\left(34^{\circ} 20^{\prime} \mathrm{S}, 116^{\circ} 10^{\prime} \mathrm{E}\right)$ from 2008 to 2016. The main study area comprised 58-70 territories, in which $>99 \%$ of the adult birds were individually color banded. Those territories were checked at least fortnightly for group composition and survival throughout the breeding season (October-January), and once a nest was located, this was monitored biweekly. Nestlings were blood sampled when at least 2 days old to determine their sex and parentage, using seven or eight hypervariable microsatellite markers, as described in Brouwer et al. (2011). We use November 1 (peak breeding season) as the census date to determine the presence and status of adults in the population. Unsuitable habitat (farmland) bounds $88 \%$ of the border of the reserve, but three narrow corridors lead away from the reserve, allowing for dispersal to the surrounding state forests (Brouwer et al. 2014a). From 2009 onward, in each year, between 50 and 220 territories in the areas surrounding the main study area (up to a $2-\mathrm{km}$ radius) were monitored and checked for dispersers. Long-distance dispersal is extremely rare (median distance $=150 \mathrm{~m}$ ), indicating that our estimates of survival are unlikely to be underestimated as a result of missed emigration. For more details on field methods, see Brouwer et al. (2011).

SFW data were collected at the Australian National Botanic Gardens in Canberra $\left(35^{\circ} 16^{\prime} \mathrm{S}, 149^{\circ} 06^{\prime} \mathrm{E}\right)$ from 1993 to 2014. The study area comprised 55-90 territories, in which $>99 \%$ of the adult birds were individually color banded. During the breeding season (September-March), the complete nesting history and performance of offspring were determined by daily census. Nestlings were blood sampled when 8 days old to determine their sex and parentage, using seven or eight hypervariable microsatellite markers (Double et al. 1997). We use November 15 (peak breeding season, when all young females have either obtained a vacancy or died) as the census date to determine the presence and status of adults in the population. Territories surrounding the study area were checked opportunistically for the presence of dispersers. Note that because of obligate dispersal, female fledgling survival in SFWs cannot be estimated accurately and represents local survival (and thus might be underestimated, as typical for nonclosed populations). However, we believe that this underestimation will not be strong; furthermore, we have no evidence that dispersal varies with group size. For more details on field methods, see Cockburn et al. (2003). Data are deposited in the Data Archiving and Networked Services EASY digital repository https://doi.org/10.17026/dans-2cf-ybgc (Brouwer et al. 2019) and the Dryad Digital Repository https://doi .org/10.5061/dryad.92rv6bq (Brouwer et al. 2020).

In both species, starvation of nestlings is negligible, but nest predation is high ( $70 \%)$. Females renest after failure, and RWFWs can initiate as many as four clutches (with one to three eggs each; Lejeune et al. 2016), but only in exceptional cases do they rear two broods to independence in a season (Russell and Rowley 2000). The longer breeding season of SFWs means that females of this species can initiate up to eight clutches (with one to five eggs each) per season and raise three broods to independence (Cockburn et al. 2016). Note that we compare group size effects in two populations of two related species and that we discuss any differences in the context of differences in life history among these species. However, we note that these life-history differences may be due to species differences in evolutionary history as well as environmental differences among the two study sites (e.g., rainfall; van de Pol et al. 2013).

\section{Statistical Analysis of Fitness Components}

We determined six fitness components: group productivity (number of fledglings produced per group per breeding season), within-group parentage (probability of paternity/ maternity within their own group), extragroup paternity (number of offspring sired by a male in another social group), offspring sex ratio, juvenile and adult survival, probability of obtaining dominant status, and probability of increasing/decreasing group size in the next year (table A1; tables A1, A2 are available online). Reproduction was split into within-group and extragroup parentage components, 
as group members can parasitize the reproductive success of the dominant breeding pair (Double and Cockburn 2003; Brouwer et al. 2011). Furthermore, group members can reduce constraints for dominant males to gain paternity outside of the social group (extragroup paternity; Mulder et al. 1994; Brouwer et al. 2017).

For each of the fitness components, we fitted a generalized linear mixed model to the data by using the R statistical language (R Development Core Team 2017) and package lme4 (Bates et al.2015) to estimate how each of these fitness components depended on group size. For annual group productivity and number of extragroup offspring, we used a Poisson model; for all other variables, we used a binomial/ binary regression model. We fitted group size as a fixed categorical effect to obtain the maximum likelihood estimate for each level of group size for each sex and social status while including year as a random intercept to account for temporal variation. We defined group size as the number of adult group members at the time of census; group sizes consisting of six or more individuals were lumped because only $0.2 \%$ (SFWs) and $1.8 \%$ (RWFWs) of groups had seven or more individuals.

A difficulty with statistical associations between group size and fitness components such as group productivity is that determining the causality of such correlations is problematic because high-quality breeders or breeders living in high-quality territories might also produce high-quality offspring and be more likely to have larger groups because of past reproductive success (Cockburn 1998). By studying the same individuals over multiple seasons with variable group sizes, we attempt to separate whether effects are due to a within-group phenotypically plastic response rather than noncausal among-group correlations due to territory or group quality. We accounted for any among-group covariance between group size and a fitness component by including a second group size predictor variable as a linear covariate: the mean group size of the group across all years (sensu within-subject centering; Snijders and Bosker 1999; van de Pol and Wright 2009). In such a model, the original group size predictor variable is then assumed to reflect the within-group effect of group size on the response variable.

\section{RVs as an Integrated Fitness Measure}

The aim here is to quantify the effect of the group size that an individual experiences in a given year on an integrative measure of fitness. Our fitness measure should be able to incorporate all abovementioned fitness components that reflect gene contributions to future generations via offspring production as well as one's own survival (and future state) into a single metric, such that we can gain understanding on how each of these fitness components (and associated behavioral mechanisms that determine variation in these fitness components) contributes to overall effects of group size on fitness. As individuals do not stay in the same group size their entire lives, individual lifetime fitness measures (lifetime number of offspring, or $\lambda_{\text {individual }}$; McGraw and Caswell 1996) have limited value here. Furthermore, formal fitness measures, such as the rate of increase of alleles (e.g., invasion fitness), are hard to measure practically. Instead, we focused on the immediate (here, annual) fitness impacts of being in a group of a specific size in a given year by determining the contribution of individuals in a given stage class (e.g., group size) to fitness. Specifically, we used RVs that quantify the contribution of individuals of a certain stage class to the long-term population growth rate (Fisher 1930), which can be used to compare the sensitivity of fitness to events at (or behaviors affecting) different (st)ages (Goodman 1982). As such, RVs can be used to quantify the strength of selection at each stage class (and thus function as an integrated fitness proxy at each stage class) and should inform us whether behaviors that affect in which group size an individual lives are favored by evolution (with the usual phenotypic gambit assumptions; Grafen 1984).

However, for species that live in groups with kin, such as fairy wrens, indirect fitness benefits also play a role (Hamilton 1964). What the optimal group size is for individuals in such a situation can be better understood by considering the question of whether it pays for an individual to accept an additional group member. Specifically, Hamilton's rule (Hamilton 1964) states that natural selection will favor a trait or behavior when the direct cost $(C)$ of a behavior to the actor outweighs the indirect benefit $(B)$ of the behavior to the recipient, with the benefits being weighted by the relatedness $(r)$ between actor and recipient $(r B>C$, the canonical version of Hamilton's rule that assumes actor control and fitness additivity; Akçay and van Cleve 2016). The RVs can be used to estimate the direct fitness costs and indirect fitness benefits and thereby the net benefits $(r B-C)$ of the behavior of "accepting another group member," and this can be done for all types of actors (dominants, subordinates, males, females) for different group sizes. For example, the costs and benefits of accepting an additional unrelated subordinate male for a dominant male in a group size of three $\left(\mathrm{D}_{3}\right)$ can be calculated as follows. The costs will be the difference between a dominant male's RV in the current group size and his RV in a group size after accepting the new subordinate male in the group: $C_{\mathrm{D}_{3}{ }_{3}}=\mathrm{RV}_{\mathrm{D}_{\hat{\gamma} 3}}-$ $\mathrm{RV}_{\mathrm{D}{ }_{4}{ }_{4}}$. Thus, understanding how $\mathrm{RV}$ s depend on group size for a given stage class directly determines the direct costs, $C$.

Similarly, the indirect benefits for the actor (A) will be the difference in RVs between group sizes of three and four of other group members (recipients [R]; e.g., a dominant $[\mathrm{D}]$ and subordinate $[\mathrm{S}]$ female also present in the group) weighted by the relatedness between the actor and the 
recipients: $r B_{\mathrm{D} \hat{\gamma}_{3}}=\sum_{R}\left(r_{\mathrm{A}, \mathrm{R}} B_{\mathrm{R}}\right)=r_{\mathrm{D} \mathrm{D}_{\odot}}\left(\mathrm{RV}_{\mathrm{D} q_{4}}-\mathrm{RV}_{\mathrm{D} q_{3}}\right)+$ $r_{\mathrm{D}^{2} \mathrm{~S}_{\mathrm{S}}}\left(\mathrm{RV}_{\mathrm{S}_{\rho_{4}}}-\mathrm{RV}_{\mathrm{S}_{\Upsilon_{3}}}\right)$. Thus, understanding how the RVs depend on group size for a given stage class also strongly informs us about the sign (and, to some extent, the strength) of the indirect benefits $(B)$ for the decision to accept additional group members. For example, if RVs of other group members are highest at a group size of three, this implies that the indirect fitness benefits of accepting additional individuals in the group will always be negative, and thus, selection will not favor larger groups (assuming $C \geq 0$; when considering the acceptance of a related-instead of unrelated-subordinate, the situation is more complex, as the benefits also depend on the relatedness-weighted benefits to the joiner).

In conclusion, RVs determine both direct costs and indirect benefits, which together shape inclusive fitness. However, a full analysis of inclusive fitness considerations, which can only be done for certain behaviors/traits/alleles (e.g., accept or reject an additional group member), is not trivial for both theoretical (Akçay and van Cleve 2016) and practical (Bourke 2014) reasons. Here we will focus on understanding the patterns and causes of variation in RV with respect to group size, which will provide the first crucial step toward understanding the inclusive fitness of group size decisions.

\section{Calculation of $R V s$}

RVs were calculated from population matrices, which were derived from a life-cycle model. We explain the main rationale of our calculations here; the exact derivation is described in the appendix (available online). We first created a life-cycle model (fig. 1a) that describes (i) the main lifehistory stages of our study systems (dominants [D], subordinates [S], and offspring [O]) and (ii) the fitness components that determine transitions between these stages (the number of fledglings produced [group productivity] multiplied by fledging survival until the next breeding season; annual adult survival for subordinates and dominants; and transition rates [conditional on survival] among states, i.e., the probability that a fledgling, subordinate, or dominant will be a dominant the next year; this life-cycle model was extended to also describe group size structure; fig. $1 b$ ), as we expected that most fitness components depended on group size, and we could model the transition probability that a group changes size between breeding seasons, which allows for including carryover effects of group living between years into the residual RV. Next, the model was extended to include two sexes, sex-specific fitness components, and an offspring sex ratio parameter (fig. A1, available online). Finally, we split reproduction into a within-group component and an extragroup parentage component to account for the fact that promiscuity levels are group size dependent as well (appendix). We note that in our model, fitness components are lo- cally density dependent (i.e., group size effects), but we were unable to include any global density regulation, as we do not have a proper understanding yet of how this complex process acts in these species (see the appendix).

The next step was then to translate our life-cycle graph into a matrix population model, in which the matrix elements consist of the six fitness components in which we are interested. We used the statistical model estimates of each fitness component derived from the field data as input to the projection matrix, which allows - using standard matrix algebra (Caswell 2001) — calculating the RVs of individuals as a function of their status, sex, and group size (see the appendix).

To quantify the amount of uncertainty in the estimates of RVs, we performed bootstrap analyses on the uncertainty of the input parameter estimates of each individual fitness component. We generated 1,000 bootstrap values based on the regression models used to analyze the association between group size and each fitness component. The mean and standard deviation of the 1,000 bootstrap values were set to be equal, respectively, to the maximum likelihood estimate and the standard error of the parameter on the scale of the link function used in the regression (for details, see the appendix). For each bootstrap input parameter set, we calculated the corresponding RVs, and by repeating this for all bootstrap sets, we derived confidence intervals around the RVs.

It should be noted that we consider group size-driven variation in fitness components independent of the statistical $P$ value of any group size effect. The reason for this is that we deem the maximum likelihood estimates for each fitness component for a given group size class to be the best estimator we have available and thus the best to be used in the overall fitness model. Moreover, including or excluding group size-driven variation in fitness components based on an arbitrary threshold $P$ value ignores the fact that (i) there is a continuous gradient of statistical support; (ii) we expected nonlinear relationships, which are hard to fit given the limited range in group size and many possible nonlinear shapes; and (iii) the statistical power to detect group size effects will vary across fitness components (e.g., statistically small effects of group size on survival will be hard to detect but may have large biological consequences for integrated fitness). Finally, by bootstrapping from the standard errors of all parameter estimates, we directly translate all information about parameter imprecision to the RV values.

\section{Decomposing How the Effect of Group Size on Each Fitness Component Contributes to Effects on RVs}

To decompose how group size effects on specific fitness components contribute to the overall effect of group size 
a

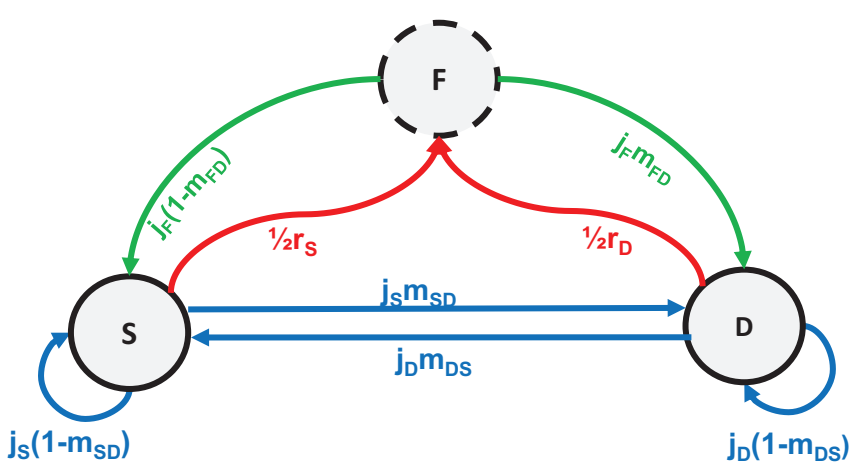

b

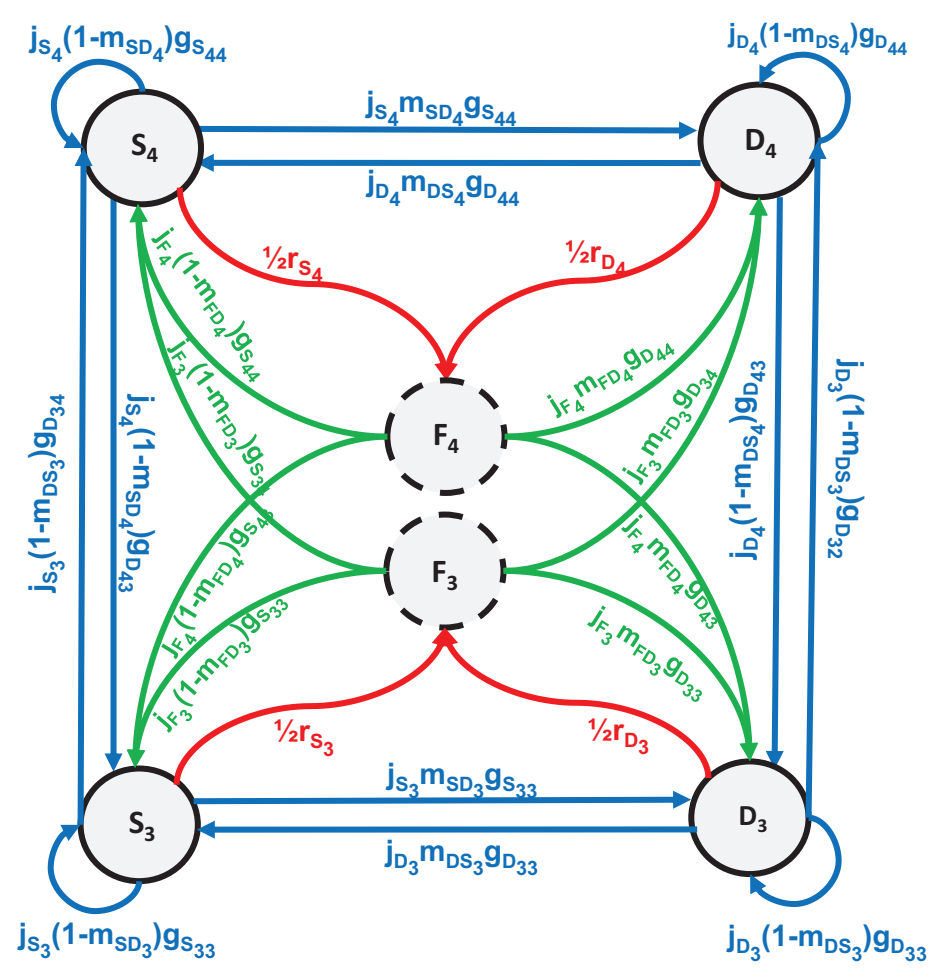

Figure 1: Simplified versions of the life-cycle graph used to model the effect of group size on the reproductive values of individuals of different sexes and statuses, in different group sizes. $a$, Arrows indicate the transition probabilities among states $(\mathrm{D}=$ dominants; $\mathrm{F}=$ fledglings; $\mathrm{S}=$ subordinates) determined by the fitness components: number of fledglings produced ( $r$ ); probability of surviving until the next breeding season for fledglings $\left(j_{\mathrm{F}}\right)$; annual adult survival for subordinates and dominants $\left(j_{\mathrm{S}}\right.$ and $j_{\mathrm{D}}$, respectively); transition rates (conditional on survival) among states $\left(m_{\mathrm{FD}}, m_{\mathrm{SD}}\right.$, and $m_{\mathrm{DS}}$; the probability that a fledgling, subordinate, or dominant will be a dominant the next year, respectively). $b$, This life cycle can be extended to model the transition probability to move from a certain group size to another $\left(g_{\mathrm{F}}, g_{\mathrm{S}}\right.$, and $g_{\mathrm{D}}$; in this example between group sizes of three and four). Note that $a$ and $b$ are simplified versions, as in our final model we included both sex differences and the range of variation in group size $(2,3,4,5$, or $6+)$ in one model (see appendix). Contributions to the next year's population via different fitness components are shown in different colors $($ red $=$ reproduction; green $=$ juvenile survival and recruitment; blue $=$ adult survival and state change).

on RV, we used a model-based thought experiment. In turn, each of the six fitness components was made independent of group size by setting the value for that fitness component to the (weighted) mean value across all group sizes (fig. 2, left panels). Each time a fitness component was made indepen- dent of group size, new RVs were calculated for each type of individual for each group size, for example, $\mathrm{RV}_{\mathrm{D}{ }^{\hat{s}}, f_{i}(\cdot)}=$ $\operatorname{RV}_{\mathrm{D} \not \partial}\left(f_{i}(\cdot), f_{\text {other }}(\mathrm{s})\right)$, with $f_{i}(\cdot)$ being the fitness component of interest that is made independent of group size and $f_{\text {other }}(\mathrm{s})$ being the other five group size-dependent fitness components 


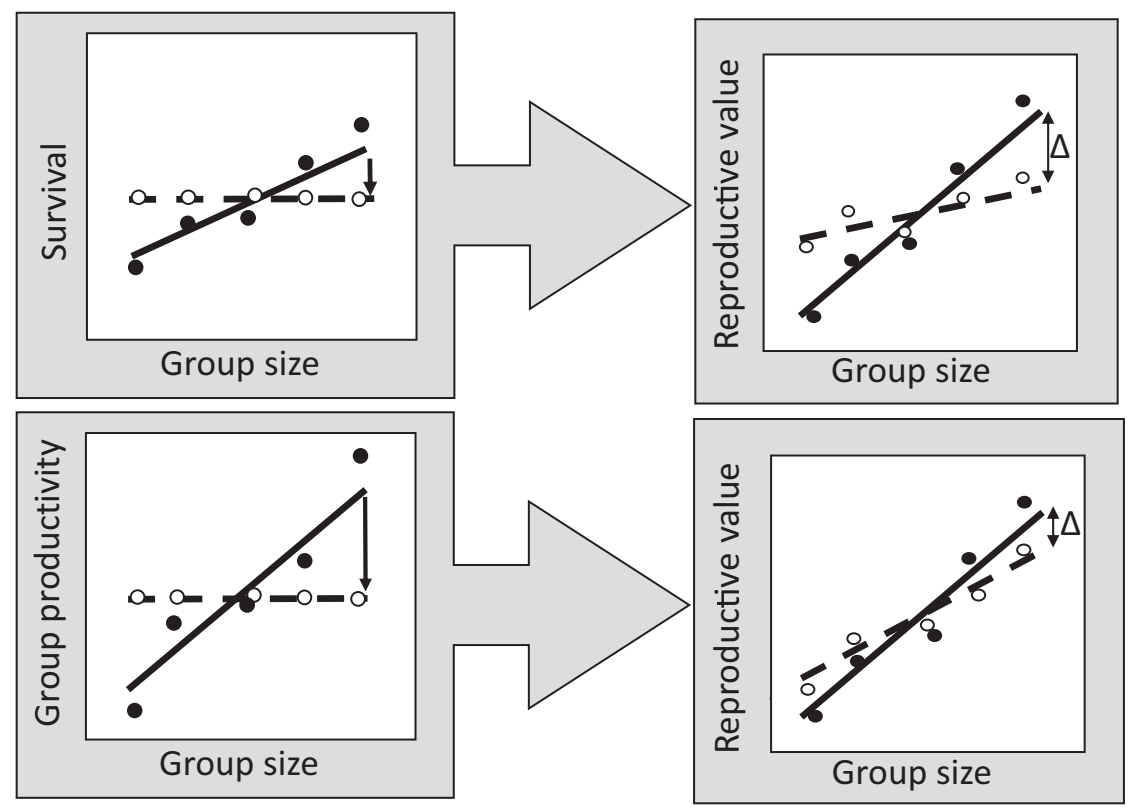

Figure 2: Graphical illustration of the decomposition analyses used to determine how group size effects on each of the six fitness components contribute to the overall effect of group size on reproductive value (RV). The left panels show how each of the fitness components (here, survival and group productivity) are made independent of group size. The right panels show the original (filled symbols) and newly calculated (open symbols) RVs, with the difference $(\Delta)$ between the two indicating the contribution of the fitness component of interest to the group size effect on RV. Note that small changes in group size effects on a fitness component (e.g., survival) can have large effects on RVs.

(with group size dependency as quantified in the empirical data). Subsequently, we calculated the difference between the group size effect on these perturbed RVs and the group size effect on the original RV estimates (i.e., $\Delta$ in fig. 2 , right panels). Specifically, we quantified the contribution $\Delta$ (in units of RV/additional group member) of the group size effect of fitness component $f_{i}$ to the group size effect on the

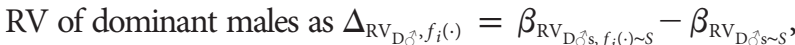
with $\beta$ being the linear regression slope coefficient of the given function.

In addition, we also calculated the combined contribution of all underlying group size effects by making all six fitness components independent of group size simulta-

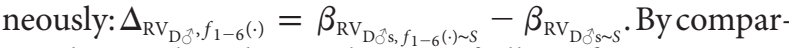
ing the combined contribution of all six fitness components to the sum of the separate contributions of all six individual fitness components $\left(\sum_{i=1}^{i=6} \Delta \mathrm{RV}_{\mathrm{D}}, f_{i}(\cdot)\right)$, we can determine whether effects of group size on fitness components affect RVs in an additive way.

As RVs initially increased from a group size of two to three and then decreased (roughly linearly) with larger group sizes (see "Results"), decomposition analyses were done in two parts to align the application of a linear decomposition to a nonlinear change with RV. We first calculated contributions for the effect of one additional group member when no (other) subordinates were present (i.e., pairs vs. a group size of three, except for subordinates that do not occur in pairs; thus, a group size of three [no other subordinate] was compared to a group size of four [one other subordinate]). Second, we calculated contributions for the effect of additional group members when looking at groups that were of size three or larger.

\section{Results}

\section{Life-History Differences between SFWs and RWFWs}

In RWFWs, group sizes were nearly 1.5 times larger than in SFWs (mean number of male subordinates per group, RWFWs vs. SFWs: 1.05 vs. 0.57; mean number of female subordinates per group, RWFWs vs. SFWs: 0.56 vs. 0.002 ; fig. $3 a, 3 b)$. Further, SFWs have a much faster life history than RWFWs. SFWs produced, on average, 2.7 times as many fledglings per group compared to RWFWs (fig. $3 c$, $3 d$ ), and they also showed much lower annual juvenile and adult survival than RWFWs (fig. $4 a, 4 b$ ), implying a twofold difference in adult life expectancy from fledging. In SFWs, females generally obtained a dominant position within their first year of life, or they died (fig. 4c). Many male fledgling SFWs were able to obtain a dominant position within their 


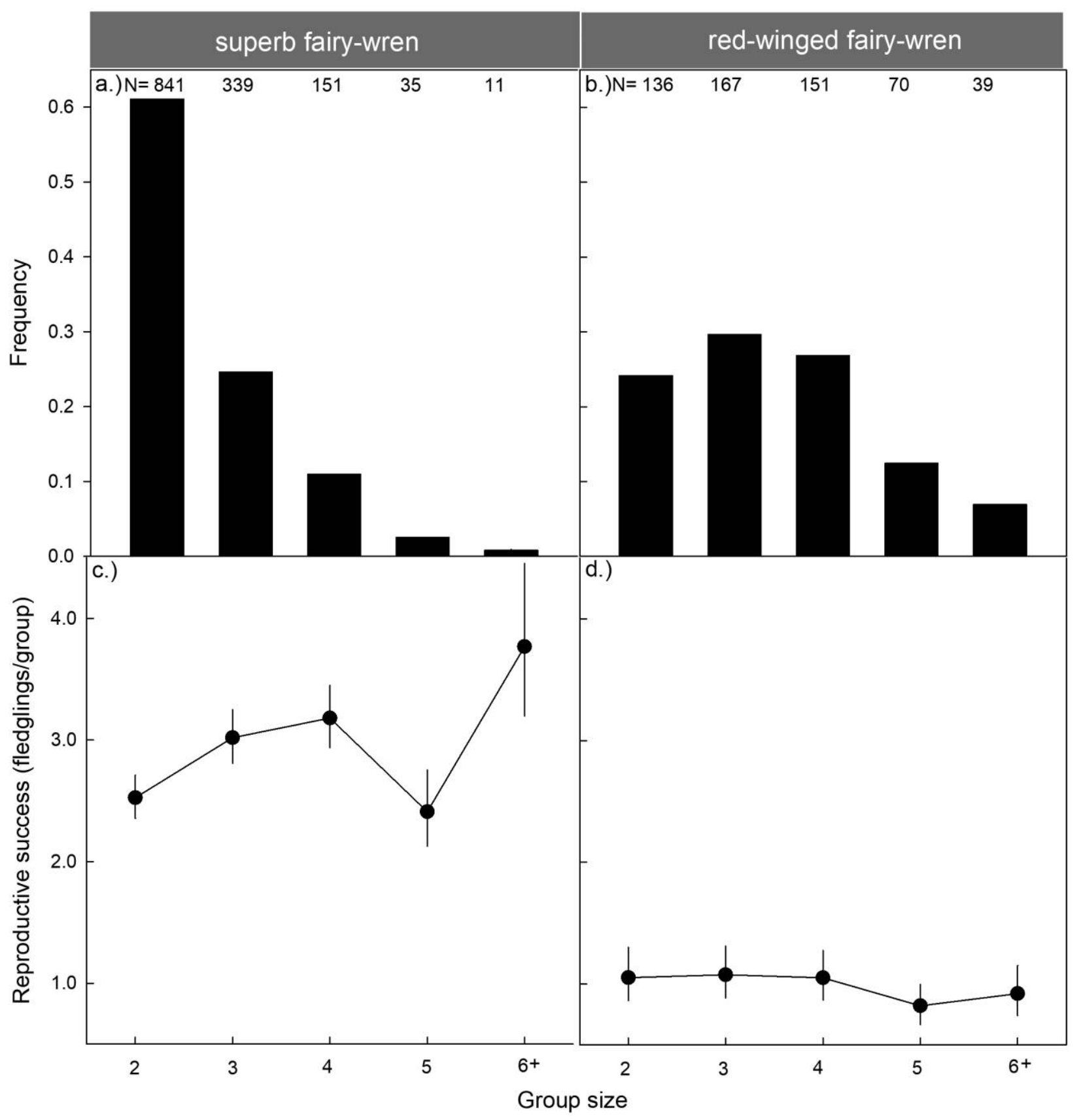

Figure 3: Relationship between group size and the frequency distribution of groups $(a, b)$ and reproductive success $(c, d)$ for superb and red-winged fairy wrens. Means are shown with their standard errors. Numbers above bars in $a$ and $b$ indicate the sample sizes (number of group years, $N$ ).

first year of life too, whereas this was extremely rare for fledgling RWFWs of either sex (fig. $4 c, 4 d$ ). In both species there was a strong positive relationship between group size from one year to the next, except for female fledglings in SFWs, which often end up in a pair after obligatory dispersal from their natal group (fig. $4 e, 4 f$ ). Patterns of genetic parent- age were remarkably similar among both species (fig. $4 g-$ $4 j$ ); most offspring are sired by extragroup males, followed by within-group dominant and subordinate males. Particularly in RWFWs, subordinate females can gain some withingroup maternity through plural breeding, which involves initiating a second nest on the territory. 


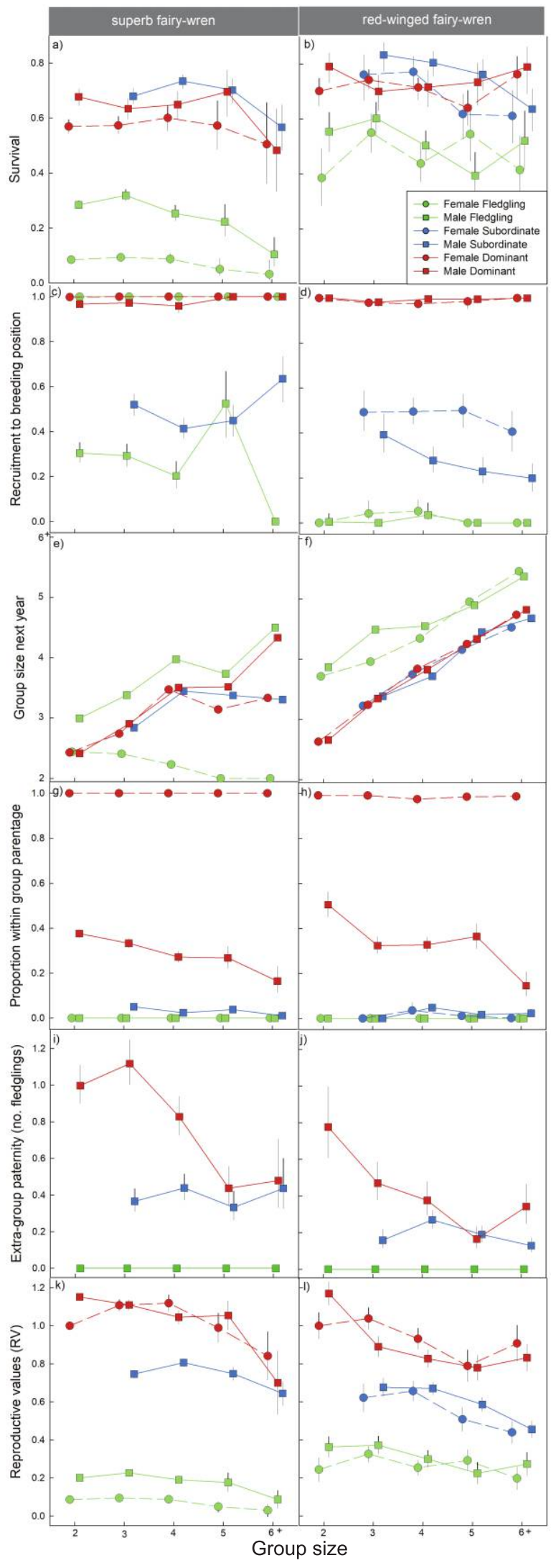

\section{Associations between Group Size and Fitness Components}

As explained in "Methods," we describe the patterns between each of the fitness components and group size, independent of their statistical significance (but more than half of the associations were significant when fitting linear or quadratic regression; see table A2). Group productivity increased with group size in SFWs but not in RWFWs (fig. $3 c, 3 d$ ). For some other fitness components, a few additional group members were beneficial, whereas larger group sizes reduced the benefits. In SFWs this was true for survival of all group members except dominant males (fig. 4a) and the number of extragroup offspring sired by dominant males (fig. 4i). RWFWs followed a somewhat similar but more erratic pattern for survival (fig. $4 b$ ).

Other fitness components were negatively associated with group size. In both species, the proportion of withingroup paternity of dominants declined with group size (fig. $4 g, 4 h$ ). Dominant males in larger groups were also less successful in siring extragroup offspring, particularly in RWFWs (fig. 4j). In both species, dominant males tended to have somewhat lower survival in the presence of a single subordinate than in pairs, whereas survival slightly increased again for larger groups (except for a group size of more than six in SFWs; however, note the large standard errors that stem from small sample sizes and further note that none of these patterns is significant; fig. $4 a, 4 b$ ). The probability that subordinates recruit to a dominant position decreased with increasing group sizes, but it increased again with very large group sizes in SFWs (fig. $4 c, 4 d$ ).

\section{Associations between Group Size and Integrated Fitness Proxy}

Aggregating the different fitness components into RVs showed the predicted nonlinear association with group size. Also as predicted, RV patterns varied among the type of individuals. Specifically, in both species, all group members except for dominant males had the highest RVs in the presence of one and, in some cases, two additional group members (fig. $4 k, 4 l$ ), while more group members reduced RVs. However, RVs of dominant males decreased in the presence of additional group members and suffered substantially in large groups (fitness reductions of $41 \%$ in SFWs and $28 \%$ in RWFWs when comparing males in groups of more than six with those living in pairs; fig. $4 k, 4 l)$.

Figure 4: Relationship between group size and maximum likelihood estimates $( \pm \mathrm{SE})$ of survival $(a, b)$, recruitment to a breeding position $(c, d)$, group size the next year $(e, f)$, proportion of within-group parentage $(g, h)$, extragroup paternity (no. fledglings; $i, j$ ), and reproductive values $(k, l)$ for superb and red-winged fairy wrens. 


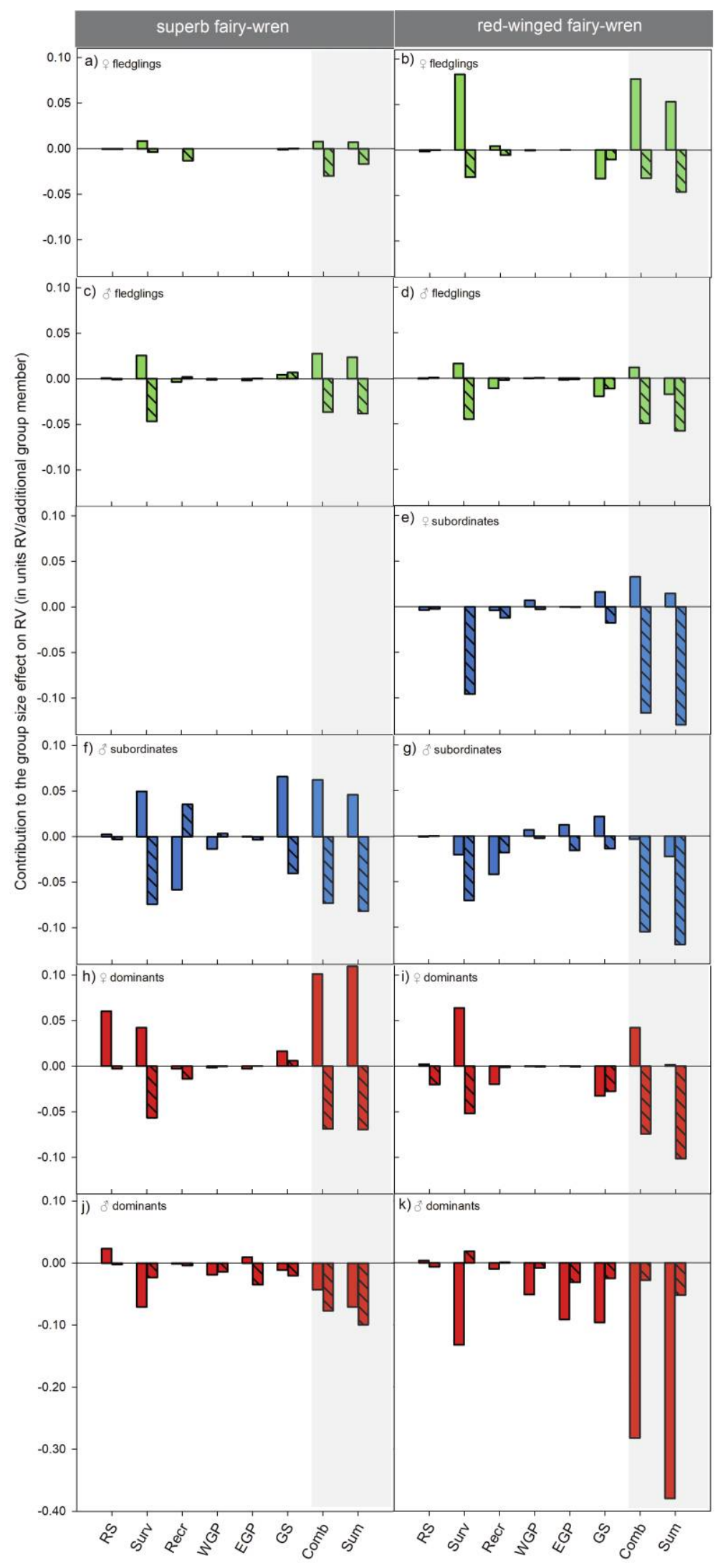

This content downloaded from 195.169.223.177 on June 12, 2020 03:53:23 AM 


\section{Relative Importance of Group Size Effects via Different Fitness Components}

For both species, decomposition analyses showed that, as predicted, the effects of group size on survival and on the carryover effects of group size from one year to the next were important determinants of group size effects on RVs (fig. 5). Only for subordinate male and dominant female SFWs living in groups without (other) subordinates was something other than the survival effect more important (fig. $5 f-5 h$, filled bars). For subordinate males, this was the group size effect on recruitment, and for dominant female SFWs this was the effect on reproductive success (fig. $5 f-5 h$, filled bars). The prediction that group size effects on reproductive success would be relatively more important for RVs in SFWs, with a faster life history compared to RWFWs, was supported for dominants, although mainly for dominant females (fig. 5h). The gain and loss of paternity both within and outside the group were relatively important for group size effects on RVs of dominant males (fig. $5 j, 5 k$ ) but less so for subordinate males (fig. $5 f, 5 g$ ).

Finally, in both species the combined contribution of group size effects via all six fitness components was typically different than the sum of the separate contributions of all six individual fitness components on RV (i.e., the whole is not the sum of its parts; fig. 5, Comb vs. Sum). This difference indicates that the group size effect on one fitness component is not independent of that on another fitness component and thus that costs and benefits of group living on RVs are nonadditive. In general, if group size negatively affected RVs, the sum of the contributions was more negative than the combined effect of group size via the different fitness components on RV (fig. 5), suggesting that group size effects on different fitness components dampen each other. By contrast, if group size positively affected RVs, the sum of the contributions tended to be less positive than the combined effects of group size (fig. 5), suggesting that group size effects on different fitness components intensify each other.

\section{Discussion}

Group living has many concurrent costs and benefits. However, a framework to integrate various fitness components had not been previously applied to this question, and therefore, it has proven difficult to determine how fitness varies with group size. Here we integrated six key fitness components into RVs and determined how these RVs varied among group members and sizes in two congeneric species that have a similar ecology but differ in some key aspects of life history. As predicted, several fitness components, as well as RV, varied nonlinearly with group size. The remarkably similar patterns in how RVs varied with group size suggest that the same behavioral mechanisms are important in both species. Furthermore, there was not much support for our prediction that RVs of the species with a faster life history would be more sensitive to effects of group size on reproductive success. For both species, the costs of additional group members on survival were most important for RVs, and this was amplified through carryover effects of group size between years (i.e., large groups suffer survival costs and are likely to do so the next year as well). In both species, RVs of most group members were highest in small groups (of size two or three), and larger group sizes reduced RVs, whereas RVs of dominant males were highest in the absence of subordinates. These results thus suggest that for most group members there are both direct costs and negative indirect fitness benefits of accepting additional unrelated group members. Finally, as predicted, we found that how group size affects RVs varied among different types of individuals, suggesting that group members might have a conflict of interest over the optimal group size.

\section{Cooperation and Competition among Group Members}

How several fitness components and RVs varied with group size was quite similar among both species, despite the fact that RWFWs had nearly 1.5 times larger group sizes, 2 -fold higher life expectancy, and 2.7-fold lower annual reproductive success. Our findings thus suggest that in both species, to a large extent, the same underlying demographic (and, thereby, likely behavioral) mechanisms are important in determining how our integrated fitness measure varies with group size and is independent of life history. All group members, except dominant males, had higher RVs in the presence of one or two additional group members. Both species are cooperative breeders with subordinates assisting in raising offspring, and previous work has already shown that this allows for load lightening in offspring-provisioning behavior (Green et al. 1995; Brouwer et al. 2014b). Living in groups might also result in thermoregulatory benefits during roosting (Hatchwell et al. 2009), which could have positive effects

Figure 5: Contribution to the group size effect on reproductive values (i.e., $\Delta$; fig. 2, right panels) for six fitness components shown for the effect of an additional group member when no (other) subordinates were present (solid bars) and for a group size of more than three (hatched bars) for superb and red-winged fairy wrens of different sexes and statuses. The six components are reproductive success (RS), survival (Surv), recruitment to a breeding position (Recr), within-group parentage (WGP), extragroup paternity (EGP), and carryover effects of group size (GS). The combined (Comb) and the summed (Sum) effect of the change in RV of the six fitness components are also shown, with the difference indicating the degree to which the combined effects are not additive. 


\section{The American Naturalist}

on survival, particularly because recent work in RWFWs suggests that changes in body condition underlie climaterelated mortality (Gardner et al. 2017, 2018).

Interestingly, despite the initial direct benefit of living with an additional group member, our results showed that the direct costs overwhelm the direct benefits in larger groups (RVs declined for larger group sizes; fig. 4). Negative effects of group size are commonly reported in groupliving species, where larger groups mean larger home ranges and thus less efficient foraging (Chapman and Chapman 2000). In contrast, additional group members in cooperative breeders usually mean more assistance in raising offspring, and relatively few empirical studies have shown that living in a large group can be costly (but see Cockburn et al. 2003; Allainé and Theuriau 2004; Brouwer et al. 2006; Sparkman et al. 2011). We found that the costs of living in larger groups were the result of higher competition for dominant positions and reduced survival, possibly due to increased competition for food. These direct costs (and benefits) of group size might vary among years or habitats. For example, in colony-living cliff swallows (Petrochelidon pyrrhonota), larger colonies survived better in cooler, wetter years (Brown et al. 2016). Future work investigating spatiotemporal fluctuations in group size effects could shed light on whether costs and benefits of group living vary with environmental conditions.

As predicted, how our fitness proxy depends on group size varied among individuals of different statuses. Notably, most group members had higher RVs in the presence of subordinates, while dominant males had lower RVs in the presence of subordinates. Because of increased competition, dominants lost both within-group and extragroup paternity success, consistent with the idea that the presence of subordinates liberates female dominants from constraints on gaining extragroup paternity (Mulder et al. 1994; Brouwer et al. 2017) and subordinates parasitizing the reproductive success of dominant males (Double and Cockburn 2003). This shows the significance of considering the genetic offspring when calculating costs and benefits of group size. At the same time, this increased competition might explain why dominant males but not females tended to have lower survival in the presence of a subordinate compared to males in pairs. Male participation in sexual competition requires costly elevated testosterone titers, and dominants have higher testosterone levels for longer periods of time than subordinates (Peters et al. 2001). Dominants thus seem to have different interests with respect to raising future group members, which suggests that there may be intragroup conflict over the optimal group size. Nevertheless, some of the loss of paternity will be gained by related subordinates and thus will partly be compensated via indirect fitness benefits. Future work calculating the inclusive fitness for different strategies (e.g., leave or stay in the group) will have to shed light on the relative importance of indirect fitness costs and benefits, and our framework provides a crucial step in doing so.

\section{Relative Importance of Group Size Effects on Different Fitness Components}

Decomposition analyses allowed us to determine how RVs changed as a result of group size effects, via underlying effects on each of the six different fitness components. The marginal—and, for most types of individuals, statistically nonsignificant-benefit of an additional group member on survival was one of the most important determinants of RVs in both species. Previous studies have emphasized the expectation that group size effects on survival may be biologically most important while statistically hardest to detect (Meade et al. 2010), and our study provides a first quantitative confirmation of this prediction. This result gives weight to the idea that the current focus in the literature on reproductive success may paint a nonrepresentative picture of the overall fitness patterns and that integrating group size effects across multiple fitness components is a research priority.

The consistent importance of the survival pathway contradicts our prediction that effects on survival are more important in a slow-life-history species and on reproductive success in a fast life history. However, although SFWs had a faster life history compared to RWFWs, it can still be considered relatively slow compared to that of Northern Hemisphere species, which usually have much lower life expectancies (annual $P_{\text {survival }}<50 \%$; Peach et al. 2001). Furthermore, the lack of importance of group size effects on reproductive success other than for female dominant SFWs (in RWFWs reproductive success did not vary with group size) might not be surprising, since subordinates only rarely sire offspring in their natal group and the high extragroup paternity rates mean that many dominant males sire offspring in other groups, which are thus unaffected by their own group size.

Strikingly, the carryover effects of group size on RVs were one of the main determinants of RVs. Although we hypothesized that such a relationship could be expected in group-living species, where group size mainly depends on the number of offspring from the previous season and on the survival of all group members, we have, for the first time, quantitatively shown that there are long-term fitness consequences of being in a group of a certain size. Surprisingly, this aspect has received little attention in studies of the costs and benefits of group living, whereas for individuals living in viscous populations, choosing the right group size will have not only important current but also future fitness consequences. 
Importantly, we showed that group size effects on different fitness components are not additive. This may not be surprising given the highly complex nonlinear relationships between group size and each of the six fitness components. Furthermore, despite the importance of the carryover effects of group size, such an effect can be important only in the presence of another strong cost or benefit, as otherwise there would be nothing to carry over. Nonadditivity thus provides another reason why fitness integration is important when considering traits that might affect many different fitness components simultaneously.

\section{Conclusion}

Our study shows that there can be many costs and benefits affecting different fitness components, suggesting that many different behavioral mechanisms play a role. These results mirror the wider literature on group living that has presented many different behavioral mechanisms (see the introduction). Our consideration of many fitness components integrated into RVs that are amenable to subsequent decomposition analysis provides much-needed insights into the key behavioral mechanisms shaping the direct costs and benefits of group living in fairy wrens. It helped us see the forest for the trees, as the role of survival costs and carryover effects of group size appear to be crucial. Conducting such attribution studies more widely will be important if we are to synthesize the relative importance of the myriad group size costs and benefits currently reported in the literature, and our study provides a quantitative analytical framework to do so. Furthermore, our approach allows for conducting subsequent inclusive fitness considerations (Hamilton 1964) and thereby provides the groundwork for future studies that aim to understand what demographic and behavioral mechanisms favor the evolution of cooperation or cause intragroup conflict.

\section{Acknowledgments}

The Western Australian Department of Biodiversity Conservation and Attractions (DBCA) and the Australian Government National Parks and Wildlife Service gave permission for fieldwork and sampling, and the Australian National University Animal Experimentation Committee licensed our field research. The Australian Bird and Bat Banding Scheme gave permission for color banding. We thank Adrian and Julia Wayne and other staff of the DBCA Science division in Manjimup and John Angus and Karen and Michael Keely for logistical support and hospitality. We are grateful to numerous students and assistants who helped in the field, in particular Helen Osmond and Marina Louter. We thank Christa Mateman for doing part of the genotyping. We would also like to thank the associate ed- itor for his helpful insights that greatly improved the quality of the manuscript. Work was supported by a Rubicon fellowship of the Netherlands Organisation for Scientific Research (NWO825.08.003) awarded to L.B. and by fellowships and grants from the Australian Research Council awarded to L.B. (DE130100174), A.C. (DP0451018, DP1092565), and M.v.d.P. (FT120100204).

\section{Literature Cited}

Akçay, E., and J. Van Cleve. 2016. There is no fitness but fitness, and the lineage is its bearer. Philosophical Transactions of the Roval Societv B 371:20150085.

Allainé, D., and F. Theuriau. 2004. Is there an optimal number of helpers in Alpine marmot family groups? Behavioral Ecology 15:916-924.

Bates, D., M. Mächler, B. Bolker, and S. Walker. 2015. Fitting linear mixed-effects models using lme4. Journal of Statistical Software 67:1-48.

Bourke, A. F. G. 2014. Hamilton's rule and the causes of social evolution. Philosophical Transactions of the Roval Society B 369:20130362.

Brouwer, L., A. Cockburn, and M. van de Pol. 2019. Data from: Integrating fitness components reveals that survival costs outweigh other benefits and costs of group living in two closely related species. Data Archiving and Networked Services EASY Digital Repository, https://doi.org/10.17026/dans-2cf-ybgc.

2020. Data from: Integrating fitness components reveals that survival costs outweigh other benefits and costs of group living in two closely related species. American Naturalist, Dryad Digital Repository, https://doi.org/10.5061/dryad.92rv6bq.

Brouwer, L., D. S. Richardson, C. Eikenaar, and J. Komdeur. 2006. The role of group size and environmental factors on survival in a cooperatively breeding tropical passerine. Iournal of Animal Ecology 75:1321-1329.

Brouwer, L., M. van de Pol, E. Atema, and A. Cockburn. 2011. Strategic promiscuity helps avoid inbreeding at multiple levels in a cooperative breeder where both sexes are philopatric. Molecular Ecology 20:4796-4807.

Brouwer, L., M. van de Pol, G. Bain, D. T. Baldassarre, L. C. Brooker, M. G. Brooker, D. Colombelli-Négrel, et al. 2017. Multiple hypotheses explain variation in extra-pair paternity at different levels in a single bird family. Molecular Ecology 26:67176729.

Brouwer, L., M. van de Pol, and A. Cockburn. 2014a. Habitat geometry does not affect levels of extrapair paternity in an extremely unfaithful fairy-wren. Behavioral Ecology 25:531-537.

. 2014b. The role of social environment on parental care: offspring benefit more from the presence of female than male helpers. Iournal of Animal Ecology 83:491-503.

Brown, C. R., M. B. Brown, E. A. Roche, V. A. O'Brien, and C. E. Page. 2016. Fluctuating survival selection explains variation in avian group size. Proceedings of the National Academy of Sciences of the USA 113:5113-5118.

Brown, J. L. 1987. Helping and communal breeding in birds. Princeton University Press, Princeton, NJ.

Caswell, H. 1982. Optimal life histories and the maximization of reproductive value: a general theorem for complex life cycles. Ecology 63:1218-1222. 
- 2001. Matrix population models: construction, analysis and interpretation. Vol. 2. Sinauer, Sunderland, MA.

Chapman, C. A., and L. J. Chapman. 2000. Determinants of group size in primates: the importance of travel costs. Pages 24-42 in S. Boinski and P. A. Garber, eds. On the move: how and why animals travel in groups. University of Chicago Press, Chicago.

Clutton-Brock, T. H. 1988. Reproductive success: studies of individual variation in contrasting breeding systems. University of Chicago Press, Chicago.

Clutton-Brock, T. H., S. J. Hodge, G. Spong, A. F. Russell, N. R. Jordan, N. C. Bennett, L. L. Sharpe, et al. 2006. Intrasexual competition and sexual selection in cooperative mammals. Nature 444:1065-1068.

Cockburn, A. 1998. Evolution of helping behavior in cooperatively breeding birds. Annual Review of Ecology and Systematics 29:141-177.

Cockburn, A., L. Brouwer, N. Margraf, H. L. Osmond, and M. van de Pol. 2016. Superb fairy-wrens: making the worst of a good job. Pages 133-149 in W. D. Koenig and J. L. Dickinson, eds. Cooperative breeding in vertebrates. Cambridge University Press, Cambridge.

Cockburn, A., H. L. Osmond, R. A. Mulder, M. C. Double, and D. J. Green. 2008. Demography of male reproductive queues in cooperatively breeding superb fairy-wrens Malurus cyaneus. Iournal of Animal Ecology 77:297-304.

Cockburn, A., H. L. Osmond, R. A. Mulder, D. J. Green, and M. C. Double. 2003. Divorce, dispersal and incest avoidance in the cooperatively breeding superb fairy-wren Malurus cyaneus. Journal of Animal Ecology 72:189-202.

Double, M. C., and A. Cockburn. 2003. Subordinate superb fairywrens (Malurus cyaneus) parasitize the reproductive success of attractive dominant males. Proceedings of the Royal Society B 270:379-384.

Double, M. C., D. Dawson, T. Burke, and A. Cockburn. 1997. Finding the fathers in the least faithful bird: a microsatellite-based genotyping system for the superb fairy-wren Malurus cyaneus. Molecular Ecology 6:691-693.

Ebensperger, L. A., D. S. Rivera, and L. D. Hayes. 2012. Direct fitness of group living mammals varies with breeding strategy, climate and fitness estimates: sociality and direct fitness in mammals. Journal of Animal Ecology 81:1013-1023.

Emlen, S. T., and P. H. Wrege. 1991. Breeding biology of whitefronted bee-eaters at Nakuru: the influence of helpers on breeder fitness. Journal of Animal Ecology 60:309-326.

Fisher, R. A. 1930. The genetical theory of natural selection. Clarendon, Oxford.

. 1958. The genetical theory of natural selection. 2 ed. Dover, New York.

Gardner, J. L., E. Rowley, P. de Rebeira, A. de Rebeira, and L. Brouwer. 2017. Effects of extreme weather on two sympatric Australian passerine bird species. Philosophical Transactions of the Roval Society B 372:20160148.

Gardner, J. L., E. Rowley, P. de Rebeira, A. de Rebeira, and L. Brouwer. 2018. Associations between changing climate and body condition over decades in two southern hemisphere passerine birds. Climate Change Responses 5:2.

Gilbert, C., D. McCafferty, Y. Le Maho, J.-M. Martrette, S. Giroud, S. Blanc, and A. Ancel. 2009. One for all and all for one: the energetic benefits of huddling in endotherms. Biological Reviews 85:545-569.
Goodman, D. 1982. Optimal life histories, optimal notation, and the value of reproductive value. American Naturalist 119:803-823.

Grafen, A. 1984. Natural selection, kin selection and group selection. Pages 62-84 in J. R. Krebs and N. B. Davies, eds. Behavioral ecology: an evolutionary approach. Blackwell, Oxford.

Green, D. J., A. Cockburn, M. L. Hall, H. L. Osmond, and P. O. Dunn. 1995. Increased opportunities for cuckoldry may be why dominant male fairy-wrens tolerate helpers. Proceedings of the Royal Society B 262:297-303.

Hamilton, W. D. 1964. The genetical evolution of social behaviour I and II. Journal of Theoretical Biology 7:1-52.

- 1971. Geometry for the selfish herd. Journal of Theoretical Biology 31:295-311.

Hatchwell, B. J., S. P. Sharp, M. Simeoni, and A. McGowan. 2009. Factors influencing overnight loss of body mass in the communal roosts of a social bird. Functional Ecology 23:367-372.

Heg, D., E. Jutzeler, D. Bonfils, and J. S. Mitchell. 2008. Group composition affects male reproductive partitioning in a cooperatively breeding cichlid. Molecular Ecology 17:4359-4370.

Horn, H. S., and D. I. Rubenstein. 1984. Behavioral adaptations and life history. Pages 279-298 in J. R. Krebs and N. B. Davies, eds. Behavioral ecology: an evolutionary approach. Blackwell, Oxford.

Huchard, E., and G. Cowlishaw. 2011. Female-female aggression around mating: an extra cost of sociality in a multimale primate society. Behavioral Ecology 22:1003-1011.

Koenig, W. D., and J. L. Dickinson, eds. 2016. Cooperative breeding in vertebrates: studies of ecology, evolution, and behavior. Cambridge University Press, Cambridge.

Koenig, W. D., and E. L. Walters. 2011. Brooding, provisioning, and compensatory care in the cooperatively breeding acorn woodpecker. Behavioral Ecology 23:181-190.

Lejeune, L., M. van de Pol, A. Cockburn, M. Louter, and L. Brouwer. 2016. Male and female helper effects on maternal investment and adult survival in red-winged fairy-wrens. Behavioral Ecology 27:1841-1850.

Majolo, B., A. de Bortoli Vizioli, and G. Schino. 2008. Costs and benefits of group living in primates: group size effects on behaviour and demography. Animal Behaviour 76:1235-1247.

Markham, A. C., L. R. Gesquiere, S. C. Alberts, and J. Altmann. 2015. Optimal group size in a highly social mammal. Proceedings of the National Academy of Sciences of the USA 112:14882-14887.

McGraw, J. B., and H. Caswell. 1996. Estimation of individual fitness from life-history data. American Naturalist 147:47-64.

Meade, J., K.-B. Nam, A. P. Beckerman, and B. J. Hatchwell. 2010. Consequences of "load-lightening" for future indirect fitness gains by helpers in a cooperatively breeding bird. Journal of Animal Ecology 79:529-537.

Mulder, R. A., P. O. Dunn, A. Cockburn, K. A. Lazenbycohen, and M. J. Howell. 1994. Helpers liberate female fairy-wrens from constraints on extra-pair mate choice. Proceedings of the Roval Society B 255:223-229.

Mumme, R. L., W. D. Koenig, and F. L. W. Ratnieks. 1989. Helping behaviour, reproductive value, and the future component of indirect fitness. Animal Behaviour 38:331-343.

Newton, I. 1989. Lifetime reproduction in birds. Academic Press, London.

- 1992. Experiments on the limitation of bird numbers by territorial behaviour. Biological Reviews 67:129-173.

Packer, C. L., L. Herbst, A. E. Pusey, J. D. Bygott, S. J. Cairns, J. P. Hanby, and M. Borgerhoff-Mulder. 1988. Reproductive success 
of lions. Pages 363-383 in T. H. Clutton-Brock, ed. Reproductive success. University of Chicago Press, Chicago.

Paquet, M., C. Doutrelant, B. J. Hatchwell, C. N. Spottiswoode, and R. Covas. 2015. Antagonistic effect of helpers on breeding male and female survival in a cooperatively breeding bird. Lournal of Animal Ecology 84: 1354-1362.

Peach, W. J., D. B. Hanmer, and T. B. Oatley. 2001. Do southern African songbirds live longer than their European counterparts? Oikos 93:235-249.

Peters, A., L. B. Astheimer, and A. Cockburn. 2001. The annual testosterone profile in cooperatively breeding superb fairy-wrens, Malurus cyaneus, reflects their extreme infidelity. Behavioural Ecology and Sociobiology 50:519-527.

R Development Core Team. 2017. R: a language and environment for statistical computing. R Foundation for Statistical Computing, Vienna.

Rowley, I., and E. Russell. 1997. Fairy-wrens and grasswrens. Oxford University Press, Oxford.

Rowley, I., E. Russell, R. Brown, and M. Brown. 1988. The ecology and breeding biology of the red-winged fairy-wren Malurus elegans. Emu 88:161.

Russell, A. F., N. E. Langmore, A. Cockburn, L. B. Astheimer, and R. M. Kilner. 2007. Reduced egg investment can conceal helper effects in cooperatively breeding birds. Science 317:941-944.

Russell, E., and I. Rowley. 2000. Demography and social organisation of the red-winged fairy-wren, Malurus elegans. Australian Journal of Zoology 48:161-200.

Snijders, T. A. B., and R. J. Bosker. 1999. Multilevel analysis; an introduction to basic and advanced multilevel modeling. Sage, London.

Sorato, E., P. R. Gullett, S. C. Griffith, and A. F. Russell. 2012. Effects of predation risk on foraging behaviour and group size: adaptations in a social cooperative species. Animal Behaviour 84:823-834.

Sparkman, A. M., J. Adams, A. Beyer, T. D. Steury, L. Waits, and D. L. Murray. 2011. Helper effects on pup lifetime fitness in the cooperatively breeding red wolf (Canis rufus). Proceedings of the Roval Society B 278:1381-1389.

Stacey, P. B., and W. D. Koenig. 1990. Cooperative breeding in birds. Cambridge University Press, Cambridge.

Stander, P. E. 1992. Cooperative hunting in lions: the role of the individual. Behavioral Ecology and Sociobiology 29:445-454 https://doi.org/10.1007/BF00170175.

Stearns, S. C. 1983. The influence of size and phylogeny on patterns of covariation among life-history traits in the mammals. Oikos 41:173-187.

van de Pol, M., L. Brouwer, L. C. Brooker, M. G. Brooker, D. Colombelli-Négrel, M. L. Hall, N. E. Langmore, et al. 2013. Problems with using large-scale oceanic climate indices to compare climatic sensitivities across populations and species. Ecography 36:249-255.

van de Pol, M., and J. Wright. 2009. A simple method for distinguishing within- versus between-subject effects using mixed models. Animal Behaviour 77:753-758.

\section{References Cited Only in the Online Enhancements}

Cockburn, A., L. Brouwer, M. C. Double, N. Margraf, and M. van de Pol. 2013. Evolutionary origins and persistence of infidelity in Malurus: the least faithful birds. Emu 113:208-217.

Mylius, S., and O. Diekmann. 1995. On evolutionary stable life histories, optimization and the need to be specific about density dependence. Oikos 74:218-224.

Pen, I., and F. J. Weissing. 2000. Towards a unified theory of cooperative breeding: the role of ecology and life history re-examined. Proceedings of the Roval Societv B 267:2411-2418.

Taylor, P. D. 1990. Allele-frequency change in a class-structured population. American Naturalist 135:95-106.

Associate Editor: Erol Akçay Editor: Alice A. Winn

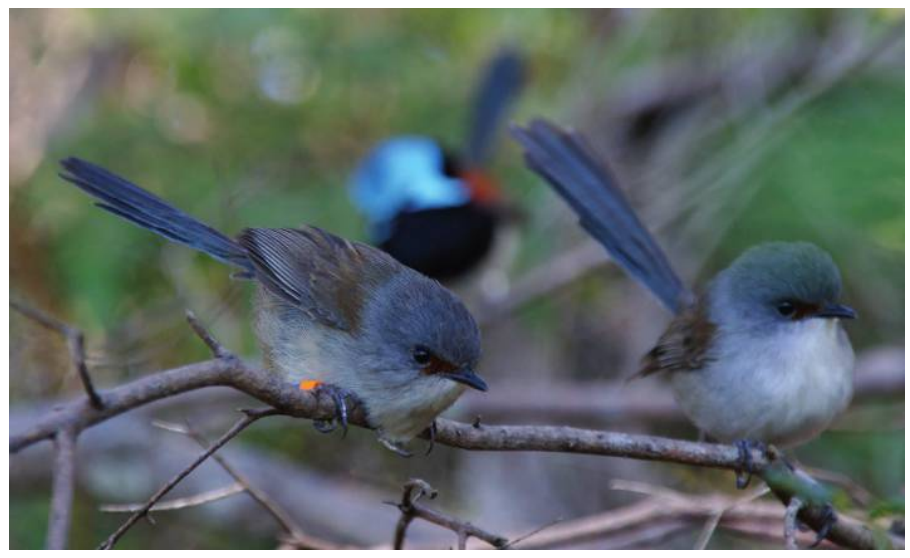

A family of red-winged fairy wrens (Malurus elegans). Photo credit: Lyanne Brouwer. 